\title{
Demonstration of Mycobacterium kansasii species heterogeneity by the amplification of the 16S-23S spacer region
}

\author{
Y. ABED, C. BOLLET* and P. DE MICCO \\ Laboratoire de Microbiologie et d'Hygiène Hospitalière, Hôpital Salvator, 249 Bld Sainte-Marguerite, \\ Marseille 13009, France
}

\begin{abstract}
Summary. Amplification of the region separating the genes coding for the two rRNA species $16 \mathrm{~S}$ and $23 \mathrm{~S}$ was performed with 56 strains of several mycobacterial species, including 21 clinical isolates of Mycobacterium kansasii and the $M$. kansasii type strain ATCC 12478 . On the basis of PCR product profiles, the previously suggested heterogeneity of $M$. kansasii species was confirmed. Three subgroups were identified; members of the first subgroup showed the same PCR profile as the reference strain. Different profiles were obtained for the two other subgroups. Amplification of the $16 \mathrm{~S}-23 \mathrm{~S}$ spacer is rapid and simple and, consequently, may be a helpful tool for identification and characterisation of $M$. kansasii isolates in epidemiological analysis.
\end{abstract}

\section{Introduction}

Mycobacterium kansasii is among the clinically important non-tuberculous mycobacteria. It is an opportunist pathogen which has been identified as an agent of disease in nearly all parts of the world. ${ }^{1}$ In association with AIDS, disseminated disease caused by $M$. kansasii has been shown to occur with increasing frequency. ${ }^{2,3}$ The homogeneity of this species has been questioned recently. The existence of a genetic subspecies was suggested on the basis of sequence differences in the 16S rRNA gene ${ }^{4}$ as well as the presence of an insertion sequence-like element in one of these subspecies. ${ }^{5}$

This study examined the heterogeneity of $M$. kansasii species on the basis of PCR profiles obtained by the amplification of $16 \mathrm{~S}-23 \mathrm{~S}$ spacer region.

\section{Materials and methods}

\section{Mvcobacterial strains}

Fifty-six mycobacterial strains were used in this study: $22 \mathrm{M}$. kansasii; $12 \mathrm{M}$. tuberculosis; $10 \mathrm{M}$. avium; five $M$. gordonae; four $M$. abscessus and three $M$. fortuitum (table). Other than the reference strains, all isolates were originally from patient specimens cultured on Lowenstein-Jensen medium and identified to species level by standard biochemical methods.
DNA extraction and amplification of $16 S-23 S$ spacer region

Mycobacterial DNA was isolated by the Chelex technique as described previously. ${ }^{6}$ DNA concentration was determined spectrophotometrically. In the amplification reaction, $25 \mathrm{ng}$ of DNA were used. PCR was performed as follows: a $50-\mu \mathrm{l}$ volume of reaction mixture containing $50 \mathrm{mM} \mathrm{KCl}, 10 \mathrm{mM}$ Tris- $\mathrm{HCl}$ (pH 8.8), $1.5 \mathrm{mM} \mathrm{MgCl}_{2}$, Triton X-100 0.1\%, $100 \mu \mathrm{M}$ of each deoxynucleotide triphosphate (Boehringer Mannheim, Mannheim Germany), 62.5 ng of each of two oligonucleotide primers G1 (5' GAAGTCGTAACAAGG 3') and L1 (5' CAAGGCATCCACCGT $\left.3^{\prime}\right)$ previously selected, ${ }^{7}$ and 1.3 units of Taq DNA polymerase (Perkin Elmer, Norwalk, CT, USA) was subjected to 25 amplification cycles of denaturation at $94^{\circ} \mathrm{C}$ for $1 \mathrm{~min}$, annealing at $55^{\circ} \mathrm{C}$ for $7 \mathrm{~min}$ and extension at $72^{\circ} \mathrm{C}$ for $2 \mathrm{~min}$. After the last cycle, samples were maintained at $72^{\circ} \mathrm{C}$ for $7 \mathrm{~min}$. A $5-\mu \mathrm{l}$ volume of the PCR product was electrophoresed in acrylamide-bis (29:1) 4\% gel with half-strength TBE $(0.025 \mathrm{M}$ Tris, $0.5 \mathrm{~mm}$ EDTA, $0.025 \mathrm{M}$ boric acid) as running buffer. The gels were stained with ethidium bromide and visualised with a UV transilluminator.

\section{Evaluation of PCR product band sizes}

Photographed gels were scanned and analysed by the Bio Image Whole Band System, Scanner 3+ (Millipore). Band sizes were calculated automatically. DNA fragments of known sizes were loaded under the 
Table. Mycobacterial strains used in this study

\begin{tabular}{lc}
\hline \multicolumn{1}{c}{ Strain } & Source \\
\hline M. kansasii & \\
ATCC 12478 & 1 \\
MKG1, MKG2, MKG3, MKG4, MKG5, MKG6 & \\
MKG7, MKG8, MKG9, MKG10, MKG11, & \\
MKG12, MKG13, MKG14, MKG15, MKG16, & \\
MKG17, MKG18, MKG19, MKG20, MKG21 & 2 \\
M. tuberculosis & \\
ATCC 27294 & 1 \\
MBE, MBKG1, MBKG2, MBKG3, MBKG4, & \\
MBKG5, MBKG6, MBKG7 & 2 \\
MKE & 3 \\
MBR & 4 \\
MTO & 5 \\
M. avium & 1 \\
ATCC 25291 & \\
MAVG1, MAVG2, MAVG3, MAVG4, MAVG5, & \\
MAVG6, MAVG7, MAVG8, MAVG9 & 2 \\
M. gordonae & \\
ATCC 14470 \\
MGOG1, MGOG2, MGOG3, MGOG4 \\
M. abscessus \\
ATCC 19977 \\
MCA1, MCA2, MCA3 \\
M. fortuitum \\
ATCC 6841 formerly TMC 1529 \\
MFR1, MFR2 & 2 \\
\end{tabular}

Source: 1, Institut Pasteur (Paris); 2, Clinical Laboratory of Salvator Hospital, Marseille (France); 3, Clinical Laboratory of Ibn Sina Hospital, Rabat (Morocco); 4, King Fayçal Hospital, Riyadh (Saudi Arabia); 5, Xin-Hua Hospital, Shanghai (People's Republic of China).

same electrophoretic conditions as PCR products and their sizes were also calculated, permitting the evaluation of the level of uncertainty in band sizes at $<1 \%$.

\section{Results and Discussion}

The profiles shown in the figure are representative of the PCR products generated by amplification of the $16 \mathrm{~S}-23 \mathrm{~S}$ spacer region for the mycobacterial species examined in this study. Reproducible patterns were obtained with $M$. tuberculosis strains, which showed fragments at 526, 471 and 333 bp and a weaker band at $303 \mathrm{bp}, M$. fortuitum strains $(588,479,453$ and two weaker bands at 1330 and $1250 \mathrm{bp}), M$. avium strains $(1340,508,468$ and $328 \mathrm{bp}), M$. gordonae strains $(523,475,357$ and $331 \mathrm{bp})$ and $M$. abscessus strains $(538,487$ and $479 \mathrm{bp}$, with a weaker band at $305 \mathrm{bp})$.

In contrast, $M$. kansasii strains showed heterogeneity in their patterns (figure, lanes C4, C5 and C6). On the basis of PCR product profiles, three subgroups were identified. The first one comprised a reference strain, ATCC 12478, and five isolates, MKG2, MKG5, MKG14, MKG17 and MKG18 which showed bands at $564,482,340,311$ and $1391 \mathrm{bp}$. The second subgroup comprised nine strains-MKG4, MKG6, MKG7, MKG8, MKG9, MKG10, MKG12, MKG13 and MKG16 - with bands at 564, 482, 340 and $400 \mathrm{bp}$, and the third subgroup comprised seven isolates-MKG1, MKG3, MKG11, MKG15, MKG19, MKG20 and MKG21 (564, 482, 340, 311 and $400 \mathrm{bp}$ ).

Spacer regions separating the three prokaryotic rRNA species $16 \mathrm{~S}, 23 \mathrm{~S}$ and $5 \mathrm{~S}$ are characterised by a high degree of sequence and length variation at both genus and species levels. This diversity is mainly due to variation in the number and type of tRNA sequences found within the spacers. ${ }^{8,9}$ The amplification of the $16 \mathrm{~S}-23 \mathrm{~S}$ spacer region has been used to identify several genera and species of pathogenic bacteria including Staphylococcus, Listeria, Escherichia, Salmonella, Enterobacter, Proteus, Clostridium and Yersinia species. ${ }^{7}$ The results of this study show that this rapid method may provide an alternative to the long culture time required for the identification of Mycobacterium

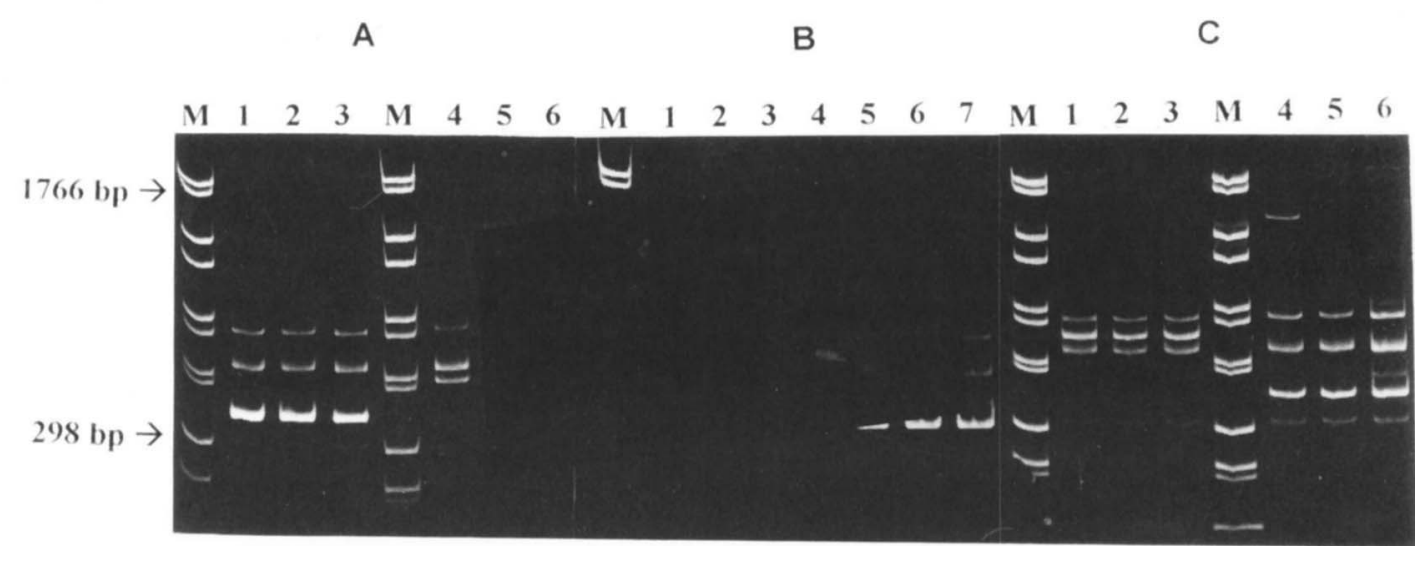

Figure. Profiles of the 16S-23S spacer region amplification products for the following mycobacterial genomic DNA samples: Lanes: A1, $M$. tuberculosis reference strain ATCC 27294; A2, A3, representative samples of $M$. tuberculosis isolates; A4, $M$. fortuitum reference strain ATCC 6841, formerly TMC 1529; A5, $M$. fortuitum strain MFR1; A6, $M$. fortuitum strain MFR2; B1, $M$. avium reference strain ATCC 25291; B2, B3, B4, representative samples of $M$. avium isolates; B5, $M$. gordonae reference strain ATCC 14470; B6, B7, representative samples of $M$. gordonae isolates; C1, $M$. abscessus reference strain ATCC 19977; C2, C3, representative samples of $M$. abscessus isolates; C4, represents $M$. kansasii reference strain ATCC 12478 and members of the first subgroup of $M$. kansasii isolates; C5, represents members of the second subgroup of $M$. kansasii isolates; C6, represents members of the third subgroup of $M$. kansasii isolates. Lanes $\mathbf{M}$ contained DNA mol. wt markers of the following sizes: $234,298,394,453,517,653,1033,1230,1766,2176 \mathrm{bp}$. 
spp. by conventional tests. Furthermore, by using this method, the recently reported heterogeneity of $M$. kansasii was confirmed. This PCR based method is rapid and simple and, consequently, may be a helpful tool for identification and differentiation of $M$. kansasii in epidemiological analysis, particularly in urgent situations.

\section{References}

1. Wolinsky E. Nontuberculous mycobacteria and associated diseases. Am Rev Respir Dis 1979; 119: 107-159.

2. Selik RM, Starcher ET, Curran JW. Opportunistic diseases reported in AIDS patients: frequencies, associations, and trends. AIDS 1987; 1: 175-182.

3. Sherer R, Sable R. Sonnenberg $\mathbf{S}$ et al. Disseminated infection with Mycobacterium kansasii in the Acquired Immunodeficiency Syndrome. Ann Intern Med 1986; 105: 710-712.

4. Ross BC, Jackson K, Yang M, Sievers A, Dwyer B. Identification of a genetically distinct subspecies of $\mathrm{Myco}$ bacterium kansasii. J Clin Microbiol 1992; 30: 2930-2933.

5. Yang M, Ross BC, Dwyer B. Identification of an insertion sequence-like element in a subspecies of Mycobacterium kansasii. J Clin Microbiol 1993; 31 : 2074-2079.
We thank M. J. Gevaudan of the clinical laboratory of Salvator Hospital (France), X. Shen of the Second Medical University of Shanghai (People's Republic of China) and L. Sahim of the Rabat Ibn Sina Hospital (Morocco) for providing mycobacterial strains.

6. de Lambalerie X, Zandotti C, Vignoli C, Bollet C, de Micco P. A one-step microbial DNA extraction method using "Chelex 100" suitable for gene amplification. Res Microbiol 1992; 143: 785-790.

7. Jensen MA, Webster JA, Strauss N. Rapid identification of bacteria on the basis of polymerase chain reactionamplified ribosomal DNA spacer polymorphisms. Appl Environ Microbiol 1993; 59: 945-952.

8. Brosius J, Dull TJ, Sleeter DD, Noller HF. Gene organization and primary structure of a ribosomal RNA operon from Escherichia coli. J Mol Biol 1981; 148: 107-127.

9. Loughney K, Lund E, Dahlberg JE. tRNA genes are found between the 16S and 23S rRNA genes in Bacillus subtilis. Nucleic Acid Res 1982; 10: 1607-1624. 